\title{
Resistance to thyroid hormone accompanied by atrial fibrillation
}

\author{
Haruhiro Sato' ${ }^{1}$ and Yuichiro Tomita ${ }^{2,3}$ \\ 1Department of Medicine, Kanagawa Dental University, Yokosuka, Kanagawa, Japan, 2Department of Pediatrics, \\ Tokai University School of Medicine Hachioji Hospital, Hachioji, Tokyo, Japan, and 3Department of Pediatrics, Keio \\ Hachioji Clinic, Hchioji, Tokyo, Japan
}

Correspondence

should be addressed

to $\mathrm{H}$ Sato

Email

h.sato@kdu.ac.jp

\section{Summary}

Resistance to thyroid hormone (RTH), which is primarily caused by mutations in the thyroid hormone (TH) receptor beta (THRB) gene, is dominantly inherited syndrome of variable tissue hyposensitivity to TH. We herein describe a case involving a 22-year-old Japanese man with RTH and atrial fibrillation (AF) complaining of palpitation and general fatigue. Electrocardiography results revealed AF. He exhibited elevated TH levels and an inappropriately normal level of thyroidstimulating hormone (TSH). Despite being negative for anti-TSH receptor antibody, thyroid-stimulating antibody and anti-thyroperoxidase antibody, the patient was positive for anti-thyroglobulin ( $\mathrm{Tg}$ ) antibody. Genetic analysis of the THRB gene identified a missense mutation, F269L, leading to the diagnosis of RTH. Normal sinus rhythm was achieved after 1 week of oral bisoprolol fumarate $(5 \mathrm{mg} /$ day) administration. After 3 years on bisoprolol fumarate, the patient had been doing well with normal sinus rhythm, syndrome of inappropriate secretion of TSH (SITSH) and positive titer of anti-Tg antibody.

\section{Learning points:}

- Atrial fibrillation can occur in patients with RTH.

- Only a few cases have been reported on the coexistence of RTH and atrial fibrillation.

- No consensus exists regarding the management of atrial fibrillation in patients with RTH.

- Administration of bisoprolol fumarate, a beta-blocker, can ameliorate atrial fibrillation in RTH.

\section{Background}

Resistance to thyroid hormone (RTH) is a rare and usually dominantly inherited disorder characterized by high levels of circulating thyroid hormone (TH) and inappropriately normal or elevated thyroid-stimulating hormone (TSH) levels, indicating a syndrome of inappropriate secretion of TSH (SITSH). Hyposensitivities to TH in various tissues have been noted in RTH. However, untreated patients with RTH usually achieve a normal metabolic state at the expense of high circulating TH levels maintained by TSH secretion in response to hypothalamic thyrotropic hormone-releasing hormone (TRH) $(1,2)$. Kahaly et al. investigated 41 patients with RTH using echocardiography and reported cardiac features that included a higher-thannormal heart rate and shorter diastolic relaxation (3). For many parameters, patients with RTH had values that were between hyperthyroidism and healthy euthyroid control. These findings suggest that reduced cardiomyocyte sensitivity to TH may be present in patients with RTH. Furthermore, ever since Usala et al. demonstrated a linkage between RTH and mutations in the TH receptor beta (THRB) gene, THRB mutations have been identified in majority of patients with RTH (4).

Only a few cases have been reported regarding the coexistence between RTH and atrial fibrillation (AF) $(5,6)$. 
We herein describe a case involving a 22-year-old Japanese man with RTH and AF who had a heterozygous missense mutation in the THRB gene wherein the normal phenylalanine had been replaced with leucine at codon 269 (F269L).

\section{Case presentation}

A 22-year-old Japanese man was referred to our hospital for palpitation and general fatigue. Four months prior to referral, he experienced general fatigue and appetite loss. One month before visiting our hospital, he visited another local hospital due to aggravation of general fatigue and appetite loss. At that time, laboratory examination results revealed elevated levels of free triiodothyronine (FT3) and free thyroxine (FT4). He was diagnosed with hyperthyroidism due to Graves' disease and received methimazole (MMI) at $20 \mathrm{mg} /$ day. However, MMI administration did not improve his general fatigue and appetite loss. Moreover, he gradually experienced palpitations, whereas FT3 and FT4 levels did not decrease to normal ranges. He was then referred to our hospital.
The patient was $173.3 \mathrm{~cm}$ in height and weighed $55.4 \mathrm{~kg}$. He lost $10 \mathrm{~kg}$ of body weight during the 4 months before presenting to our hospital. His physical examination revealed a clear state of consciousness, blood pressure of $112 / 62 \mathrm{mmHg}$, irregular pulse rate of 72 beats/ min and body temperature of $37.0^{\circ} \mathrm{C}$. No heart murmurs, abnormal heart or lung sounds and facial or pretibial edema were noted. His thyroid was mildly enlarged and palpable. No proptosis or finger tremors were evident. The patient, as well as his parents and sister, had no medical history, including thyroid disease.

\section{Investigation}

AF was detected using electrocardiography (ECG) (Fig. 1). Complete blood cell counts and blood chemistry tests revealed elevated levels of total cholesterol and triglyceride ( $244 \mathrm{mg} / \mathrm{dL}$ and $194 \mathrm{mg} / \mathrm{dL}$, respectively). Serum TSH, FT3 and FT4 levels were $2.26 \mu \mathrm{IU} / \mathrm{mL}$ (reference range: 0.50 $5.00 \mu \mathrm{IU} / \mathrm{mL}$ ), $5.09 \mathrm{pg} / \mathrm{mL}$ (reference range: $2.30-4.00 \mathrm{pg} /$ $\mathrm{mL}$ ) and $2.09 \mathrm{ng} / \mathrm{mL}$ (reference range: 0.90-1.70 ng/ $\mathrm{mL})$, respectively. Titers of anti-TSH receptor antibody

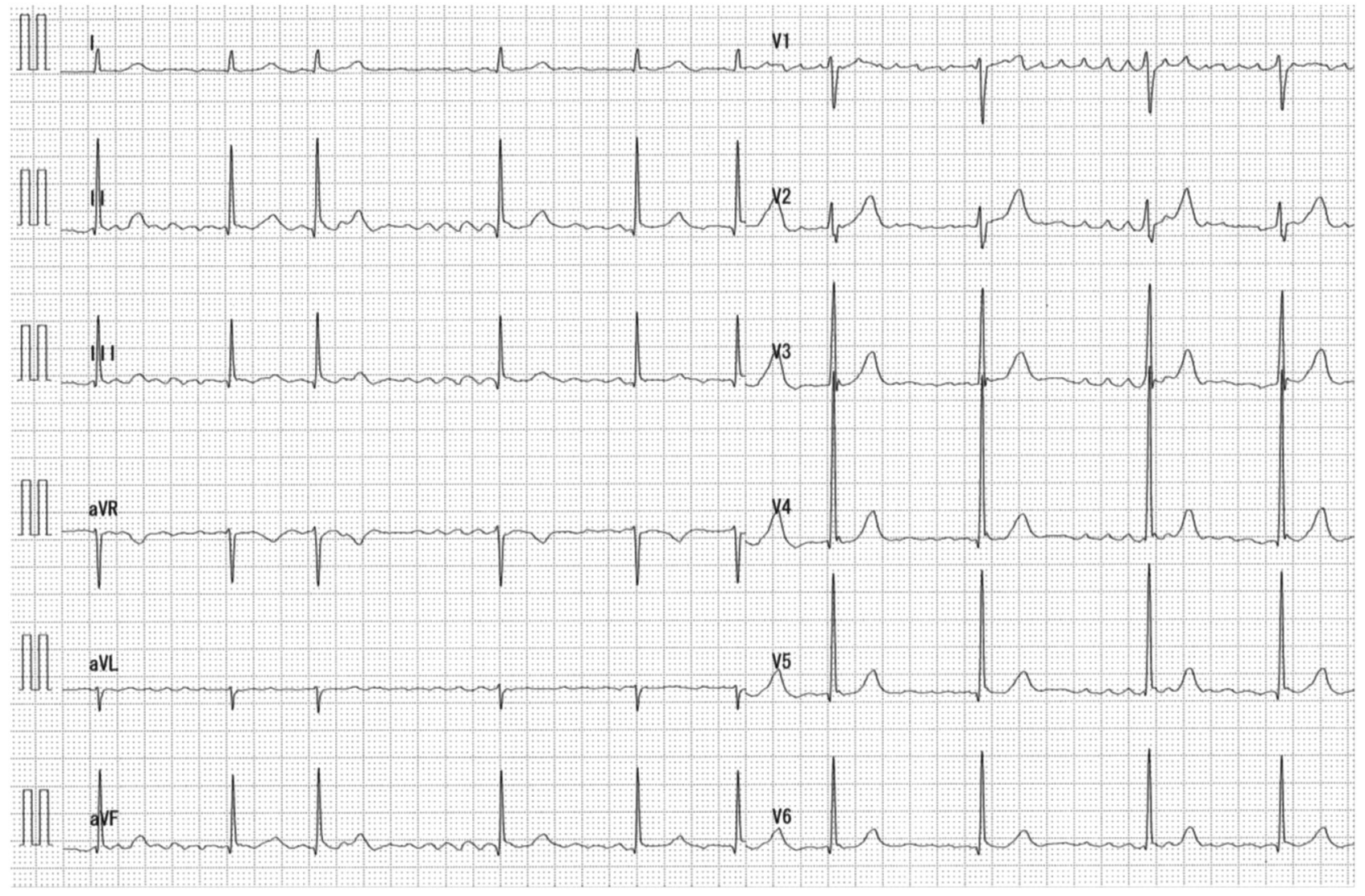

Figure 1

Atrial fibrillation as observed using electrocardiography. 
(TRAb), thyroid-stimulating antibody (TSAb) and antithyroperoxidase (TPO) antibody were negative, whereas those of anti-thyroglobulin ( $\mathrm{Tg}$ ) antibody was positive (302 IU $/ \mathrm{mL}$; reference range, $<28 \mathrm{IU} / \mathrm{mL}$ ). Such features indicated the presence of SITSH. Moreover, the TRH stimulation test showed peak TSH and prolactin values of $17.63 \mu \mathrm{IU} / \mathrm{mL}$ and $44.4 \mathrm{ng} / \mathrm{mL}$, respectively.

Ultrasonography of the thyroid showed a diffuse thyroid gland enlargement without altered echotexture of the parenchyma. A 20-min technetium-m99 pertechnetate (99mTcO4) thyroid uptake imaging study demonstrated elevated thyroid uptake (4.5\%; reference range: $0.4-3 \%$ ) and a normally shaped thyroid gland (Fig. 2). MRI showed no pituitary adenoma.

After providing written informed consent to participate in this study, the patient underwent the $T H R B$ gene analysis. The investigation was performed in accordance with the principles of the Declaration of Helsinki. This study was approved by the Institutional Review Board Committee at Tokai University School of Medicine. Blood samples were obtained from the patient, and genomic DNA was isolated from leukocytes using standard protocol. Direct sequencing of the amplified $T H R B$ was performed using the standard cycle on an automated sequencer as described elsewhere (7). Genetic analysis revealed a heterozygous point mutation of THRB, TTC to TTA at codon 269, resulting in the substitution of phenylalanine with leucine (F269L) (Fig. 3). The patient's

\section{Ant R}

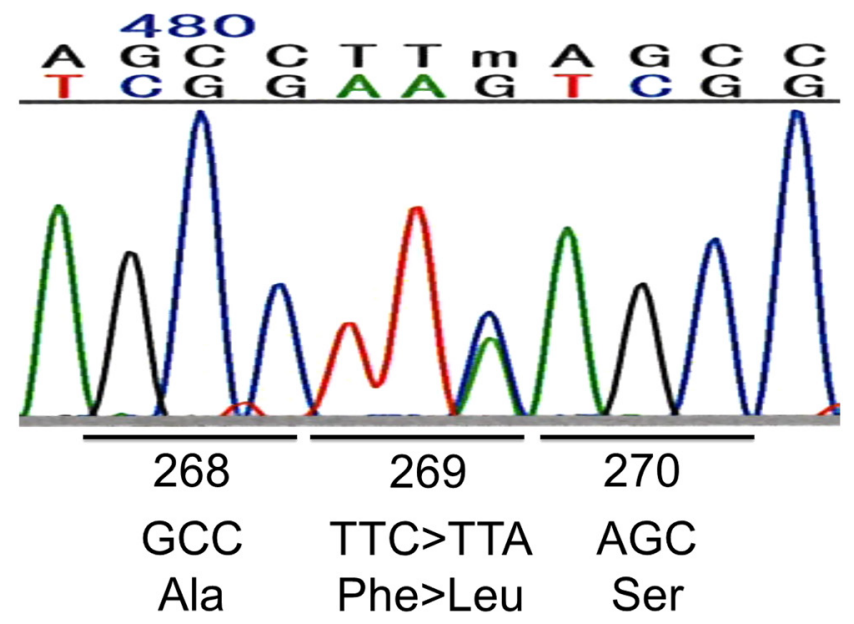

Figure 3

Sequence analysis of the THRB gene revealing a heterozygous point mutation, TTC to TTA, resulting in the substitution of phenylalanine with leucine at codon 269 (F269L).

parents and sister were not examined as they were unwilling to undergo DNA analysis.

\section{Treatment}

MMI was discontinued because of SITSH. Moreover, after consulting with cardiologists at our hospital regarding the AF experienced by the patient, beta-blocker therapy was recommended given that the onset of AF was unclear, vital signs were stable, both CHADS2 and CHA2DS2VASC scores were 0 and SITSH was observed. Oral administration of bisoprolol fumarate $(5 \mathrm{mg} /$ day $)$ was started, resulting in the amelioration of $\mathrm{AF}$ and heart rhythm correction to normal sinus rhythm 7 days after initial drug administration.

\section{Outcome and follow-up}

After 3 years on bisoprolol fumarate, the patient had been doing well with normal sinus rhythm, SITSH and positive titer of anti-Tg antibody.

\section{Discussion}

We herein describe a case involving a 22-year-old Japanese man who exhibited SITSH accompanied by AF. In addition, MRI revealed no pituitary adenoma, while the TRH stimulation test showed normal TSH response. Such findings excluded the possibility of TSH-producing pituitary adenoma. Moreover, despite being negative for titers of TRAb, TSAb and anti-TPO antibody, the patient was
Figure 2

A 20-min technetium-m99 pertechnetate $(99 \mathrm{mTCO} 4)$ thyroid uptake imaging study indicating an elevated thyroid uptake $(4.5 \%$; reference range, $0.4-3 \%$ ) and a normally shaped thyroid gland. 
positive for anti-Tg antibody. Accordingly, the THRB gene analysis showed that the patient carried a heterozygous missense mutation, F269L, which had not been previously reported. Thus, the patient had been diagnosed with RTH due to a novel THRB mutation (F269L) accompanied by AF. Codon 269 of THRB is in exon 8 (2), and functional characterization of the mutation, F269L, showed SITSH and normal response to the TRH stimulation test, which were compatible with features of other mutations of THRB $(1,2)$. It was reported that individuals with RTH due to THRB mutations have an increased likelihood of autoimmune thyroid diseases (8). Thus, positive titer of anti-Tg antibody in the patient may not be coincidental. Clinical phenotype of the patient indicated goiter, which was one of the common phenotypes in RTH $(1,2)$.

One of the clinical features presented by the patient during the first visit was AF. Similarly, Wakasaki et al. described a case involving a 60-year-old man with RTH complicated with type 2 diabetes mellitus and cardiomyopathy (5). At 53 years of age, the patient exhibited bradycardia and $\mathrm{AF}$, which were noted on ECG. He subsequently, received a permanent pacemaker, and MMI administration was initiated. Accordingly, the authors suggested that AF may occur in patients with RTH.

$\mathrm{TH}$ is involved in diverse functions, including cardiac and gastrointestinal function, regulation of skeletal growth, maturation of the central nervous system and energy homeostasis. Such actions are mediated by $\mathrm{TH}$ receptors encoded by two genes, TH receptor alpha (THRA) gene and the THRB gene, each of which undergoes alternate splicing to generate receptor subtypes (THRA1, THRB1 and THRB2) with differing tissue distributions $(9,10)$. THRA1 is predominant in cardiac and skeletal muscles, bone and gastrointestinal tract; THRB1 is most abundant in the liver and kidneys and THRB2 is discretely expressed in the hypothalamus, pituitary gland, cochlea and retina (8). Sinus tachycardia has been found to be common among patients having RTH, with some studies reporting a frequency as high as $80 \%$ (9). Accordingly, tachycardia observed in patients with RTH may reflect cardiac sensitivity to TH through intact THRA1.

In addition, reports have shown that patients with thyrotoxicosis had an AF prevalence of 1.7-28\% $(11,12)$, though the relationship between RTH and AF remained unclear. However, cardiac response to excess $\mathrm{TH}$ has been suggested to increase and cause AF given that the heart develops less resistance to TH. Majority of patients with AF develop arrhythmia for a relatively short time $(<4-8$ weeks) (12). This was consistent with the AF observed in our patient, which had ameliorated within 1 week after oral bisoprolol fumarate administration.

In conclusion, we have described a case involving a 22-year-old Japanese man with RTH, and AF who carried an F269L mutation of THRB. Accordingly, cardiac sensitivity to TH through intact THRA1 in cardiomyocytes may have been the cause of $\mathrm{AF}$ in our patient. AF had been successfully ameliorated through the administration of the beta-blocker bisoprolol fumarate.

\section{Declaration of interest}

The authors declare that there is no conflict of interest that could be perceived as prejudicing the impartiality of the research reported.

\section{Funding}

This research did not receive any specific grant from any funding agency in the public, commercial or not-for-profit sector.

\section{Patient consent}

Written informed consent for publication was obtained from the patient.

Author contribution statement

$\mathrm{H}$ Sato is a consultant endocrinologist. $\mathrm{Y}$ Tomita is a pediatric endocrinologist. $\mathrm{H}$ Sato and $\mathrm{Y}$ Tomita contributed equally to the management of this patient and the drafting of the report. $\mathrm{H}$ Sato moved to Kanagawa Dental University from Tokai University School of Medicine. $\mathrm{H}$ Sato is the corresponding author and $\mathrm{Y}$ Tomita is the author

\section{References}

1 Refetoff S, Weiss RE \& Usala SJ. The syndromes of resistance to thyroid hormone. Endocrine Reviews 199314 348-399. (https://doi. org/10.1210/edrv-14-3-348)

2 Dumitrescu AM \& Refetoff S. The syndromes of reduced sensitivity to thyroid hormone. Biochimica et Biophysica Acta 20131830 3987-4003. (https://doi.org/10.1016/j.bbagen.2012.08.005)

3 Kahaly GJ, Matthews CH, Mohr-Kahaly S, Richards CA \& Chatterjee VK. Cardiac involvement in thyroid hormone resistance. Journal of Clinical Endocrinology and Metabolism 200287 204-212. (https://doi.org/10.1210/jcem.87.1.8170)

4 Usala SJ, Bale AE, Gesundheit N, Weinberger C, Lash RW, Wondisford FE, McBride OW \& Weintraub BD. Tight linkage between the syndrome of generalized thyroid hormone resistance and the human c-erbA beta gene. Molecular Endocrinology 19882 1217-1220. (https://doi.org/10.1210/mend-2-12-1217)

5 Wakasaki H, Matsumoto M, Tamaki S, Miyata K, Yamamoto S, Minaga T, Hayashi Y, Komukai K, Imanishi T, Yamaoka H, et al. Resistance to thyroid hormone complicated with type 2 diabetes and cardiomyopathy in a patient with a TRbeta mutation. Internal Medicine 201655 3295-3299. (https://doi.org/10.2169/ internalmedicine.55.7147)

6 Alberto G, Novi RF, Scalabrino E, Trombetta A, Seardo MA, Maurino M \& Brossa C. Atrial fibrillation and mitral prolapse in a subject affected by Refetoff syndrome. Minerva Cardioangiologica 2002 50 157-160. 
7 Sasaki S, Nakamura H, Tagami T, Miyoshi Y, Nogimori T, Mitsuma T \& Imura H. Pituitary resistance to thyroid hormone associated with a base mutation in the hormone-binding domain of the human 3,5,3'-triiodothyronine receptor-beta. Journal of Clinical Endocrinology and Metabolism 199376 1254-1258. (https://doi.org/10.1210/ jcem.76.5.8496318)

8 Barkoff MS, Kocherginsky M, Anselmo J, Weiss RE \& Refetoff S. Autoimmunity in patients with resistance to thyroid hormone. Journal of Clinical Endocrinology and Metabolism 201095 3189-3193. (https://doi.org/10.1210/jc.2009-2179)

9 Lazar MA. Thyroid hormone receptors. Multiple forms, multiple possibilities. Endocrine Reviews 199314 184-193. (https://doi org/10.1210/edrv-14-2-184)
10 Beck-Peccoz P \& Chatterjee VKK. The variable clinical phenotype in thyroid hormone resistance syndrome. Thyroid 19944 225-232. (https://doi.org/10.1089/thy.1994.4.225)

11 Shimizu T, Koide S, Noh JY, Sugino K, Ito K \& Nakazawa H. Hyperthyroidism and the management of atrial fibrillation. Thyroid 200212 489-493. (https://doi. org/10.1089/105072502760143863)

12 Sawin CT, Geller A, Wolf PA, Belanger AJ, Baker E, Bacharach P, Wilson PW, Benjamin EJ \& D'Agostino RB. Low serum thyrotropin levels as a risk factor for atrial fibrillation in older persons. New England Journal of Medicine 1994331 1249-1252. (https://doi. org/10.1056/NEJM199411103311901)

Received in final form 8 August 2018

Accepted 31 August 2018 